\title{
Isochaihulactone-induced DDIT3 causes ER stress-PERK independent apoptosis in glioblastoma multiforme cells
}

\author{
Sheng-Feng Tsaí $^{1}$, Michael Tao ${ }^{2}$, Li-Ing Ho${ }^{3}$, Tzyy-Wen Chiou ${ }^{4}$, Shinn-Zong Lin ${ }^{5}$, \\ Hong-Lin Su${ }^{1}$, Horng-Jyh Harn ${ }^{6,7}$ \\ ${ }^{1}$ Department of Life Sciences, National Chung-Hsing University, Taichung, Taiwan \\ ${ }^{2}$ Department of Bioengineering, University of California, San Diego, La Jolla, California, USA \\ ${ }^{3}$ Department of Chest Medicine, Taipei Veterans General Hospital, Taipei, Taiwan, School of Medicine, National Yang-Ming \\ University, Taipei, Taiwan \\ ${ }^{4}$ Department of Life Science, Graduate Institute of Biotechnology, National Dong Hwa University, Hualien, Taiwan \\ ${ }^{5}$ Bioinnovation Center, Tzu Chi foundation, Department of Neurosurgery, Hualien Tzu Chi Hospital, Hualien, Taiwan \\ ${ }^{6}$ Bioinnovation Center, Tzu Chi Foundation, Department of Pathology, Buddhist Tzu Chi General Hospital, Hualien, Taiwan \\ ${ }^{7}$ Department of Pathology, China Medical University Hospital, Taichung, Taiwan \\ Correspondence to: Horng-Jyh Harn, email: duke_harn@yahoo.com.tw \\ Hong-Lin Su, email: suhonglin@gmail.com
}

Keywords: ER stress, glioblastoma, DDIT3, isochaihulactone, NAG-1

Received: May 11, $2016 \quad$ Accepted: November 02, 2016

Published: November 10, 2016

\section{ABSTRACT}

The endoplasmic reticulum (ER) is a major site of cellular homeostasis regulation. Under the ER stress condition, Glioblastoma multiform (GBM) cells activate the unfolded protein response. In this study, we discovered isochaihulactone, a natural compound extracted from the Chinese traditional herb Nan-Chai-Hu, which can disrupt ER homeostasis in GBM cell lines. It can induce DNA damage inducible transcript 3 (DDIT3) expression which is independent of $78 \mathrm{kDa}$ glucose-regulated protein (GRP78) and protein kinase RNA-like endoplasmic reticulum kinase (PERK) expression. Flow cytometry results revealed that isochaihulactone trigger the cell cycle arrest at $\mathrm{G} 2 / \mathrm{M}$ phase and apoptosis in GBM cells. Isochaihulactone induced DDIT3 led to the expression of NAG-1. The in vivo study showed that isochaihulactone suppressed tumor growth, and DDIT3 and Caspase 3 overexpressed in the xenograft model, which is consistent with the in vitro study. Overall, the data revealed that isochaihulactone disrupted ER homeostasis in cancer cells by increasing DDIT3 and NAG-1 expression. Our finding also provides a therapeutic strategy by using isochaihulactone for GBM treatment.

\section{INTRODUCTION}

The endoplasmic reticulum takes part in several cellular functions, including protein synthesis, calcium homeostasis, and phospholipid synthesis [1]. ER stress is always present in cells undergoing rapid proliferation, hypoxia, and therapeutic treatment [2]. During ER stress, increased levels of unfolded proteins accumulate and cause protein kinase RNA-like endoplasmic reticulum kinase (PERK), an ER stress sensor, to activate. Moreover, the other ER stress sensor, inositol-requiring enzyme $1 \alpha$ (IRE1 $\alpha$ ), also activates the translation of HSP90 chaperone family member GRP78. GRP78 is involved in the subsequent folding steps for a subset of ER client proteins, a process called the unfolded protein response (UPR) [3].
In tumor cells, ER stress may restore homeostasis and render the adjacent environment hospitable for tumor survival and growth [4]. GRP78, are highly expressed in tumor cells, and contribute to cancer progression [5]. The UPR is usually inactive in normal cells, but is widely overexpressed in many cancers, including breast cancer [6], leukemia [7], colon cancer [8], and brain cancer.

Glioblastoma multiform and anaplastic astrocytoma, the most common and severe primary gliomas in adults, are highly mobile, invasive, and difficult to resect completely through surgery. PERK, DDIT3 and GRP78 are all expressed at low levels in the normal adult brain, but GRP78 are highly express in malignant GBM cell lines [9], and in GBM patients [10]. With increased GRP78 expression, this will lead to poor survival rate. 
In recent years, researchers have begun studying the potential role of GRP78 in GBM therapy [11-13]. Tian et al. used a different strategy in which increased levels of ER stress activated DDIT3 expression directly which led to cell apoptosis [14].

Nan-Chai-Hu (Chai Hu of the South), the root of Bupleurum scorzonerifolium, is a valuable Chinese herb used in the treatment of influenza, fever, malaria, cancer, and menstrual disorders in China, Japan, and many other parts of Asia. Previous studies have reported that the compound isochaihulactone (K8), isolated from the crude acetone extract of $B$. scorzonerifolium, causes cell cycle arrest at G2/M phase in lung adenocarcinoma A549 cells, and activates non-steroidal anti-inflammatory drugactivated gene (NAG-1) expression, thereby triggering A549 cell apoptosis [15]. By using a microarray, we discovered K8 induced DDIT3. However, the relationship between DDIT3 and NAG-1 has not been determined for K8 against GBM.

In this study, we used K8 to treat GBM cell lines, and discovered that GRP78 was not activated and PERK was downregulated under K8 treatment, despite strong DDIT3 expression. Our results proved that K8 upregulate DDIT3 expression, but will not activate PERK; previous studies have proven that PERK modulates DDIT3 expression. We subsequently demonstrated that DDIT3 increased NAG-1 expression in GBM cell lines, which led to tumor cell apoptosis. Finally, K8 reduced the tumor size considerably, and increased DDIT3 and caspase- 3 expression in vivo.

\section{RESULTS}

\section{ER-Stress-related proteins are widely expressed in glioblastoma multiform cell lines}

The ER-related proteomic profile was used to compare the ER stress level in different cell lines. Each GBM cell line, 8401, 8901, U87, G2T, 131TXM, 1XM, RG2, and GL261, showed high levels of ER stress marker PERK and UPR major modulator GRP78 (Figure 1A), compared with human primary astrocyte. These levels exhibited no correlation with respect to ER stress apoptosis-related protein DDIT3 (Figure 1A). As shown in Figure 1B, quantified data revealed there are two cell lines, 8401 and G2T, with high level PERK expression but lower level of downstream protein DDIT3. Therefore, we suggest that DDIT3 expression might not depend on PERK activation. Figure 1D shows the DDIT3, PERK, and GRP78 expression profiles in 8401 and G2T, following $\mathrm{K} 8$ treatment. We discovered that the level of the ER stress modulator GRP78 did not change following K8 treatment (Figure 1F). However, with K8 treatment, PERK expression was down regulated (Figure 1E). DDIT3, was consequently strongly expressed (Figure 1G).

\section{$\mathrm{K8}$ is a potential drug that can induce glioblastoma cell death}

We tested the cytotoxicity of K8 on GBM cancer cells, 8401, G2T and astrocyte. MTT Assay tests revealed that $\mathrm{K} 8$ exerted a strong anti-proliferative effect on the GBM cell lines (Figure 2A-2C). Compared with untreated cells, K8-treated 8401 and G2T cells floated on the surface of the medium, which is a typical feature of cells undergoing apoptosis (Figure 2D).

\section{K8 causes glioblastoma cell cycle arrest and apoptosis}

To investigate the effects of $\mathrm{K} 8$ on cell cycle progression, GBM cell lines were exposed to IC50 of K8 for 0,24 and 48 hours, and the cell cycle phase distribution was analyzed using FACS (Figure 3A). The quantification of untreated 8401 cells showed that $61.8 \%$ of the cells were in the G0/G1 phase, $14.5 \%$ were in the $\mathrm{S}$ phase, and $20.4 \%$ were in the $\mathrm{G} 2 / \mathrm{M}$ phase of the cell cycle 48 hours after plating. The treatment of 8401 cells with $60 \mu \mathrm{M}$ of K8 for 48 hours increased the percentage of cells in the $\mathrm{G} 2 / \mathrm{M}$ phase to $26.6 \%$, and reduced the amount of cells in $\mathrm{G} 0 / \mathrm{G} 1$ and $\mathrm{S}$ phases to $44.1 \%$ and $15.7 \%$, respectively. The subG1 population of cells exceeded $12.5 \%$, and G2T cells treated with K8 $60 \mu \mathrm{M}$ for 48 hours, increased the percentage of cells in the G2/M phase from $22.1 \%$ to $49.6 \%$. The subG-1 population of cells also exceeded $17.3 \%$. These two cell lines went through cell cycle arrest in $\mathrm{G} 2 / \mathrm{M}$ phase under $\mathrm{K} 8$ treatment (Figure $3 \mathrm{~B}$ and $3 \mathrm{C}$ ). At the same time, we also performed an AnnexinV-PI double stain to verify that 8401 and G2T cells treated with $\mathrm{K} 8$, depending on the dose, will undergo apoptosis. Two cell lines went through apoptosis in low dosages of K8 (8401 : 12.7\%; G2T: 39.2\%) and apoptotic population increase to $37.3 \%(8401)$ and $48.1 \%$ (G2T) under K8 $80 \mu \mathrm{M}$ treatment. (Figure 3D). The statistical result showed that K8 significantly caused these two cell lines to go through apoptosis (Figure 3E). Based on FACS data, we discovered that apoptosis played a role in $\mathrm{K} 8$ induced cell death. We found that K8-induced cell death was due to caspase pathway activation. After 8401 and G2T cells were treated with K8 for 48 hours, we harvested whole cell lysates for western blotting. PARP, caspase-3, caspase-9, and caspase-7 were upregulated and Bcl-2 was downregulated, following K8 treatment (Figure 3F). Thus, K8-induced cell death in 8401 and G2T cells in the caspase-dependent pathway.

\section{Knockdown $N A G-1$ and $D D I T 3$ expression can restore 8401 cell viability}

The significantly increased DDIT3 expression played a crucial role in the investigation of the mechanism 
responsible for the K8-induced glioblastoma cell apoptosis. We used DDIT3 siRNA to knockdown DDIT3 protein expression, which restored the 8401 cell viability (Figure 4A), and inhibited apoptosis-protein-cleavedcaspase-3 expression (Figure 4B). Previous studies showed that $\mathrm{K} 8$ can also activate NAG-1 expression in lung and prostate carcinoma, but the mechanism is still unclear $[15,16]$. In this study, we showed K8 activated NAG-1 expression not only in lung and pancreatic carcinoma, but also in glioblastoma (Figure 4C). And, we used $N A G-1$ siRNA to silence NAG-1 expression, which was induced by K8 treatment. In our result, K8-induced NAG-1 expression led to 8401 cell apoptosis (Figure 4D-4E). We found 8401 and G2T cell lines had high levels of PERK, with no DDIT3 expression. Previous research showed that the PERK inhibitor GSK2606414 caused the prion-infected cell to escape when overloaded under ER stress and then undergoes apoptosis [17]. Our hypothesis is that K8 will induce DDIT3 with no PERK activation. Therefore, we used GSK 2606414 to inhibit PERK expression and treated 8401 and G2T cells with K8. Our data showed that $\mathrm{K} 8$ decrease the cell viability

A

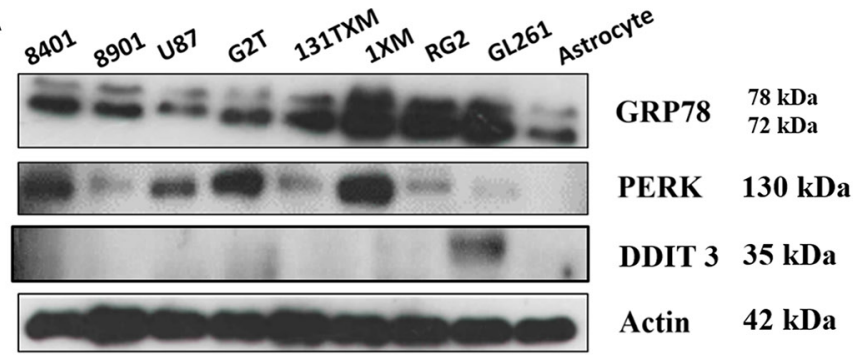

B

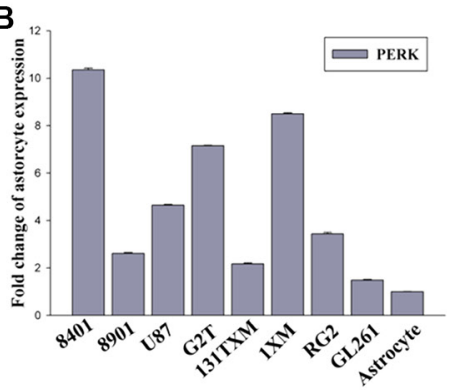

D

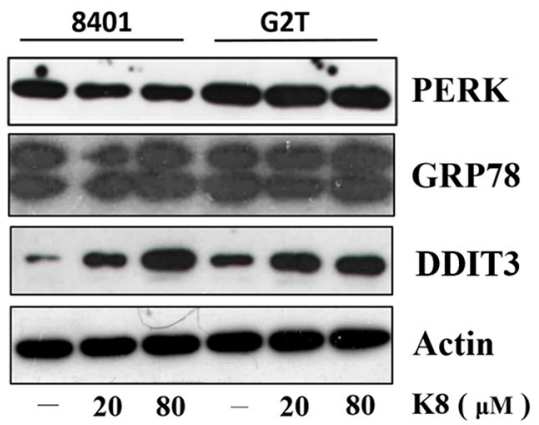

E

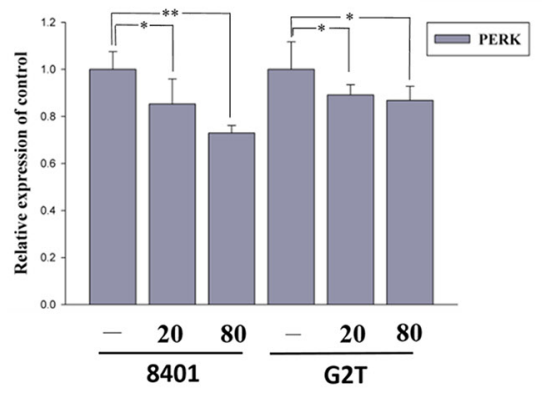

C

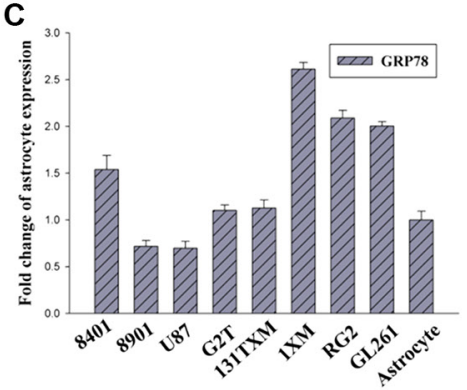

$130 \mathrm{kDa}$

$78 \mathrm{kDa}$

$72 \mathrm{kDa}$

$35 \mathrm{kDa}$

42 kDa

$\mathbf{F}$

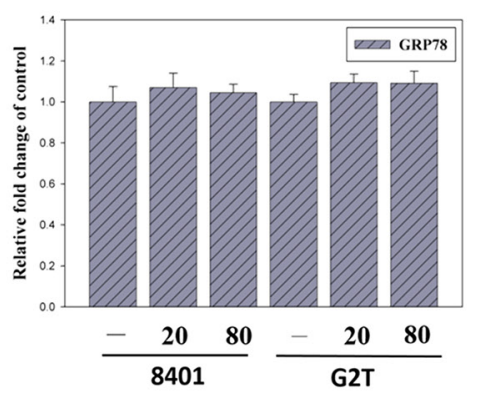

G

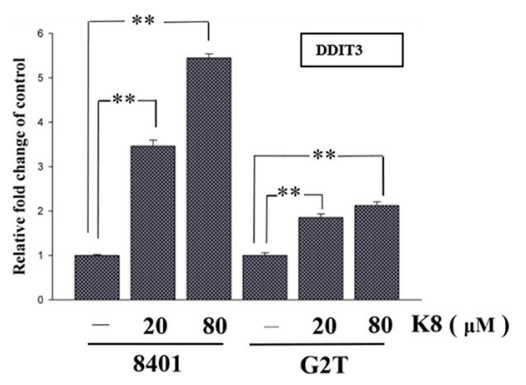

Figure 1: K8 induced DDIT3 expression in glioblastoma cell lines. (A) ER stress marker expression basal level in glioblastoma cell line. (B-C) Quantification of PERK and GRP78 expression basal level compared with astrocyte. (D) Protein expression of ER stress markers were performed by Western blotting within K8 treatment in 24 hours. (E-G) Quantification showed PERK expression downregulated, GRP78 was not affected, and there was significant increase of DDIT3 compared to the control group. Values shown are the mean \pm S.E.M. from three experiments. Level of statistical significance: $* p<0.05$, $* p<0.01$. 
of glioblastoma when used in tandem with GSK 2606414 (Figure 4F). The western blotting revealed that GSK 2606414 inhibited PERK expression; K8 with GSK 2606414 induced DDIT3 expression (Figure 4G).

\section{K8 induced DDIT3, which led to NAG1 expression, and finally, 8401 cell undergo apoptosis}

We discovered that the increase in DDIT3 gene expression was accompanied by a considerable rise in $N A G-1$ gene expression. DDIT3 siRNA was used to inhibit $D D I T 3$ gene expression, which reduced $N A G-1$ gene expression substantially, by more than $40 \%$. Although we used siRNA transfection to knockdown NAG-1 expression, K8-induced DDIT3 expression remained unchanged in the transcription level (Figure 5A) and the translation level (Figure 5B) stages. Overall, these results indicate that DDIT3 regulates NAG-1 gene expression.

\section{K8 reduces glioblastoma tumor growth and increases tumor DDIT3 expression in a xenograft model}

The xenograft model that was used to evaluate the in vivo antitumor activity of K8. Approximately $1 \times 10^{6}$ 8401 cells was injected into the back of nude mice. After the tumor grew approximately $100 \mathrm{~mm}^{3}$ in volume, the mice were randomized into 3 groups: (1) Control (vehicle), (2) Low-dose treatment $(50 \mathrm{mg} / \mathrm{kg})$, and (3) High-dose treatment $(200 \mathrm{mg} / \mathrm{kg})$. Treatment groups (6 animals each) were given daily subcutaneous injections of $\mathrm{K} 8$ for 5 days. The result revealed that K8 inhibited tumor growth at low doses, whereas K8 induced tumor regression at high doses (Figure 6A); weight loss was not observed during the experiment (Figure 6B). Additional histological examination revealed DDIT3 expression in vivo (Figure 6C). The data showed that $\mathrm{K} 8$ did not cause PERK expression; however, the DDIT3 and
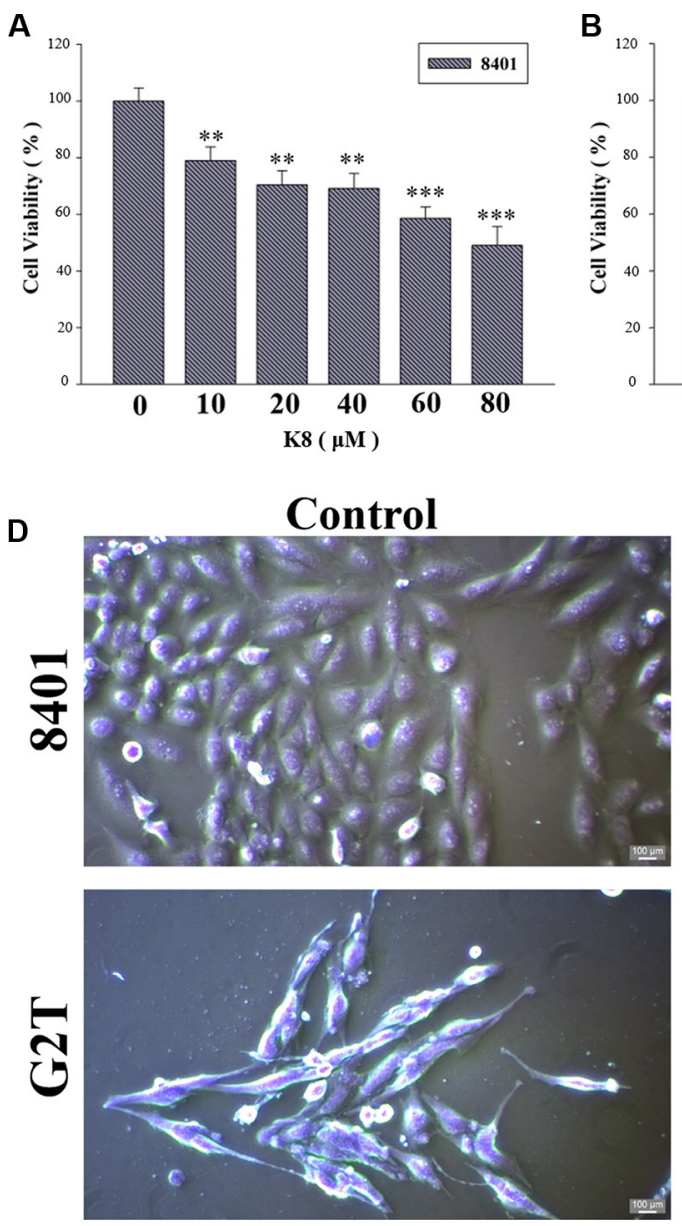
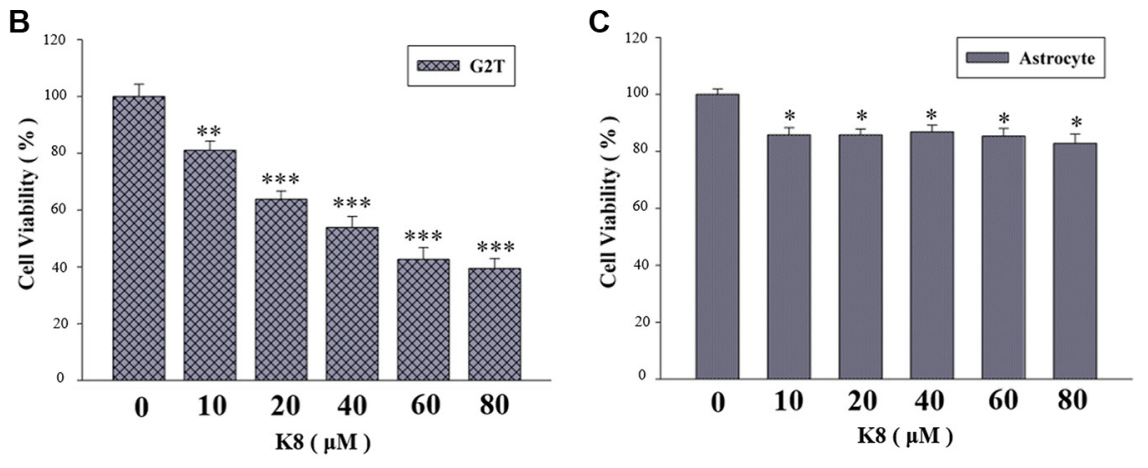

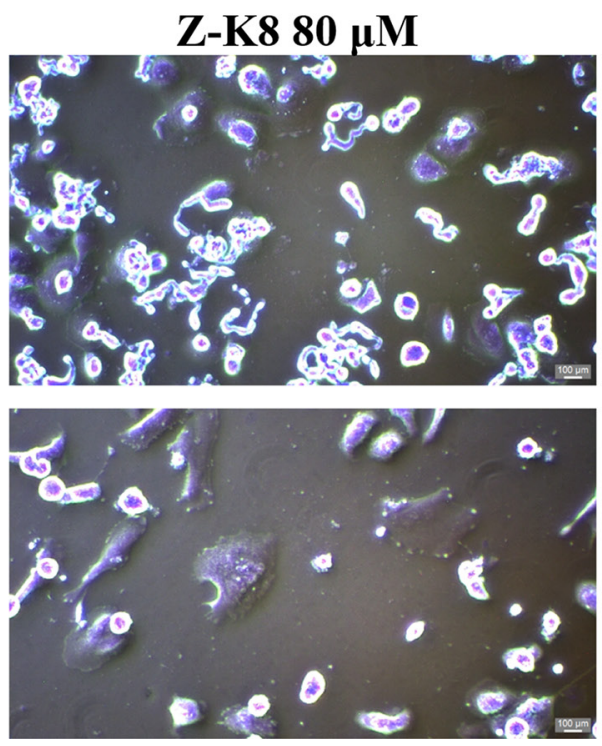

Figure 2: K8 induced cell death in glioblastoma cell lines. (A-B) Glioblastoma cancer cell lines 8401 and G2T were treated with $\mathrm{K} 8$ for $24 \mathrm{hrs}$ in a dose dependent manner. (C) Human primary astrocyte. Cell viability was detected by MTT assay. K8 reduced 8401 and G2T cell viability but does not affect astrocyte viability. Level of statistical significance: ${ }^{*} p<0.05, * * p<0.01, * * * p<0.001$. (D) Effects of morphology and chromatin change of K8 in 8401 and G2T. Morphological examination of K8-induced giant cells after cells were treated with $80 \mu \mathrm{M}$ of $\mathrm{K} 8$ for $24 \mathrm{hrs}$. 
cleaved caspase- 3 was expressed with higher doses of K8. K8-induced apoptosis is mediated by the DDIT3modulated NAG-1 apoptotic pathway (Figure 7).

\section{DISCUSSION}

Increasing evidence supports the hypothesis that ER stress is very important in many diseases, including cancer [18]. We first analyzed the ER stress conditions in various GBM cell lines. We demonstrated that GBM cells experienced ER stress with increased levels of PERK, and that GRP78 was overexpressed in these cell lines. However, DDIT3 levels were low. Under K8 treatment, GRP78 was not affected and PERK was downregluated; however, DDIT3 was upregulated. Miyake et al. found that GRP78 overexpression prevented DDIT3 expression which also inhibited cell apoptosis. Tunicamycin, an ER stress inducer, can causes ER stress overload and prevents GRP78 from functioning, thus leading cells to undergo apoptosis [19]. Many researchers have developed anti-GRP 78 drug to block the ER stress repair system which induces cancer apoptosis. $[13,20]$. The present study results revealed that DDIT3 and NAG-1 were upregulated, leading to tumor cell apoptosis with K8 treatment. Therefore, we found another pathway different from PERK-eIF2 $\alpha$ axis, where K8 induces DDIT3 expression directly.

This study is the first to report that DDIT3 regulates NAG-1 expression with K8 treatment. According to our results, DDIT3 regulates NAG-1 expression at the transcriptional level, which means that DDIT3 may alter NAG-1 mRNA expression and its stability. The ER stress induced the DDIT3-mediated cytosolic translocation of HuR and the formation of stress granules. The HuR positively affected NAG-1 gene regulation by stabilizing NAG-1 mRNA during ER stress. The NAG-1 expression is modulated at various levels, including transcriptional and post-transcriptional regulation [21]. Rottlerin,

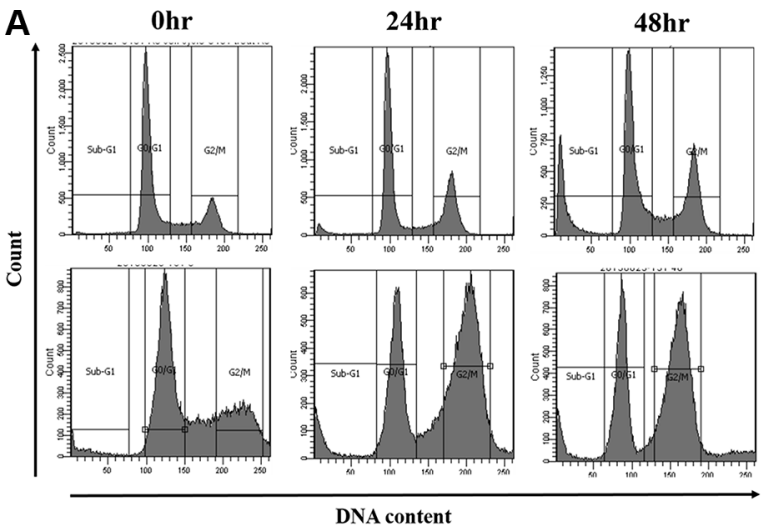

B

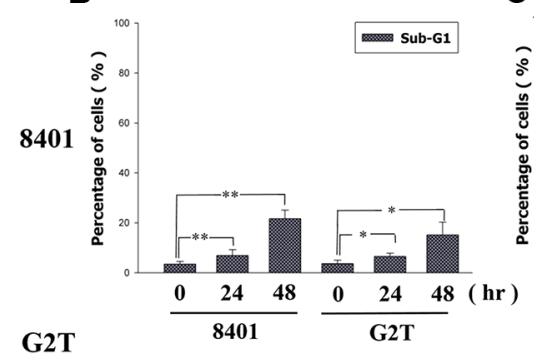

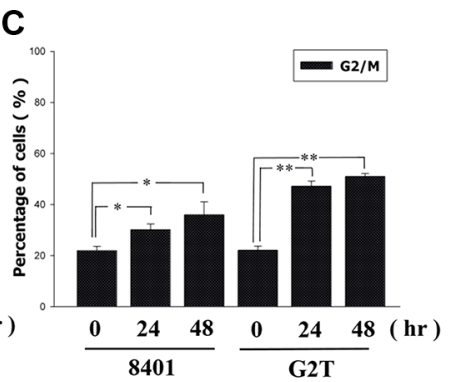

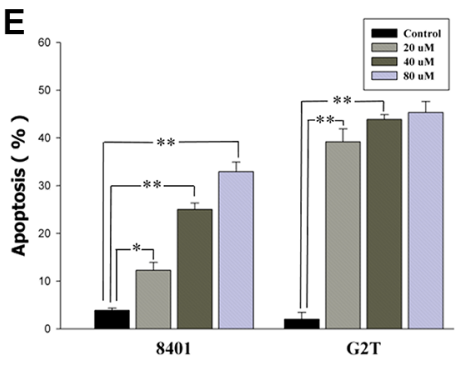

F

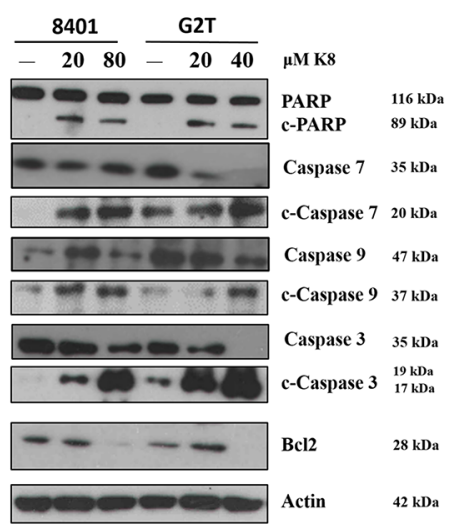

Figure 3: Effects of K8 on cell cycle and apoptosis in glioblastoma cell lines 8401 and G2T. (A) The cells were treated with $\mathrm{K} 880 \mathrm{uM}$ for $0,24,48 \mathrm{hrs}$ and analyzed for cell distribution by flow cytometry. (B-C) The subG1 and G2/M cell population were quantified. (D-E) 8401, G2T were treated with K8 20, 40, $80 \mathrm{uM}$ for $24 \mathrm{hrs}$ and then analyzed for AnnexinV-PI double stain to detect for cell apoptosis. (F) After K8 treatment of 8401 and G2T cell lines apoptosis-related protein was expressed. Experiments were carried out at least three times. Level of statistical significance: ${ }^{*} P<0.05,{ }^{*} p<0.01$. 


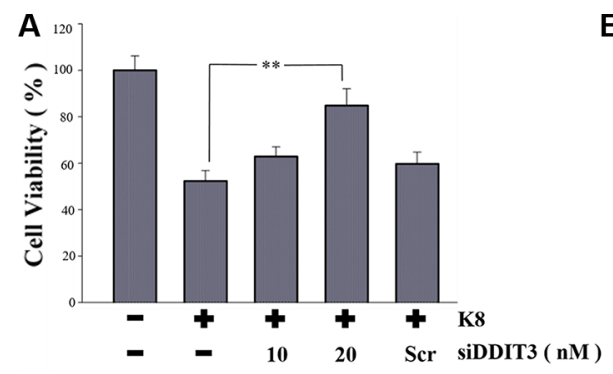

B

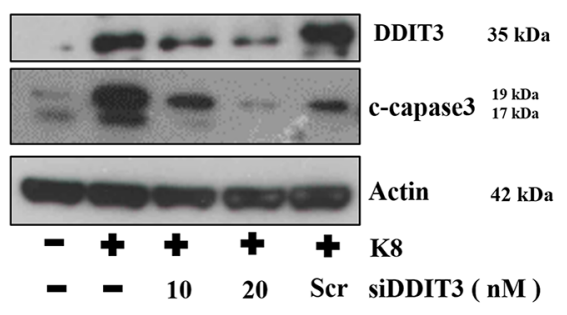

E
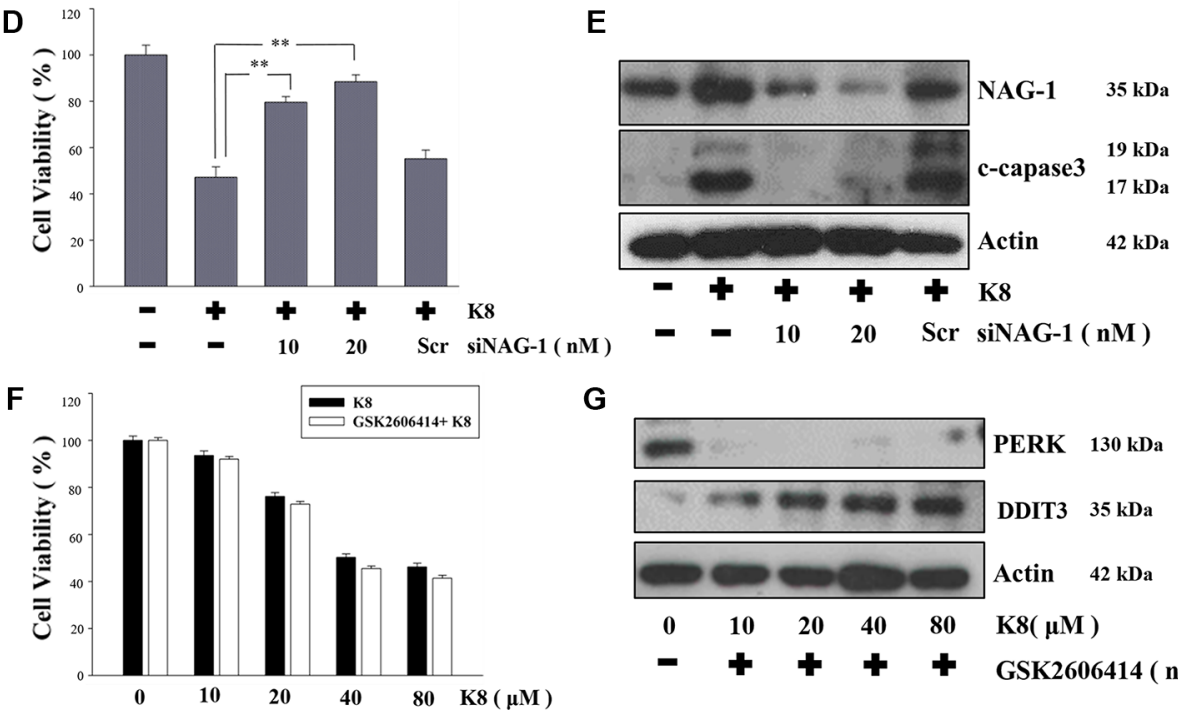

G

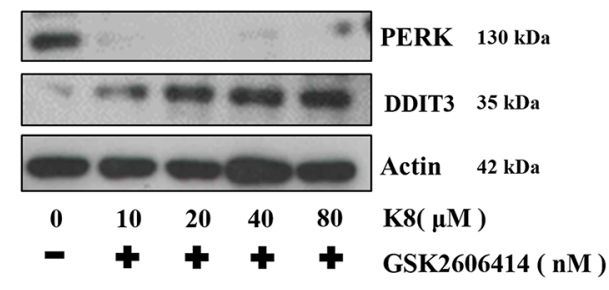

C

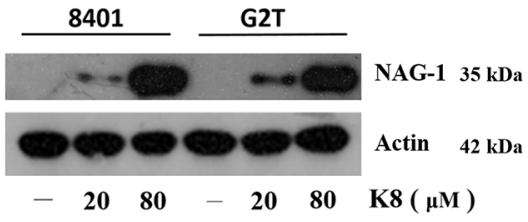

Figure 4: Effect of DDIT3, NAG-1, PERK on the induction of apoptosis by K8 in 8401. (A, D) 8401 cells were transfected with various concentrations of DDIT3 or NAG-1 siRNA (10, $20 \mathrm{nM})$ and treated with K8 for 24 hrs. (B, E) Western blot analysis were performed for DDIT3, NAG-1 and cleaved capase3. (C) K8 induced NAG-1 expression in 8401 and G2T cell lines. (F) 8401 cells were first treated with $10 \mathrm{nM}$ PERK inhibitor GSK2606414 for $24 \mathrm{hrs}$ and then treated with K8 in a dose-dependent manner. (G) Western blot analysis was performed for PERK and DDIT3. Level of statistical significance: ${ }^{*} p<0.05,{ }^{*} p<0.01$.

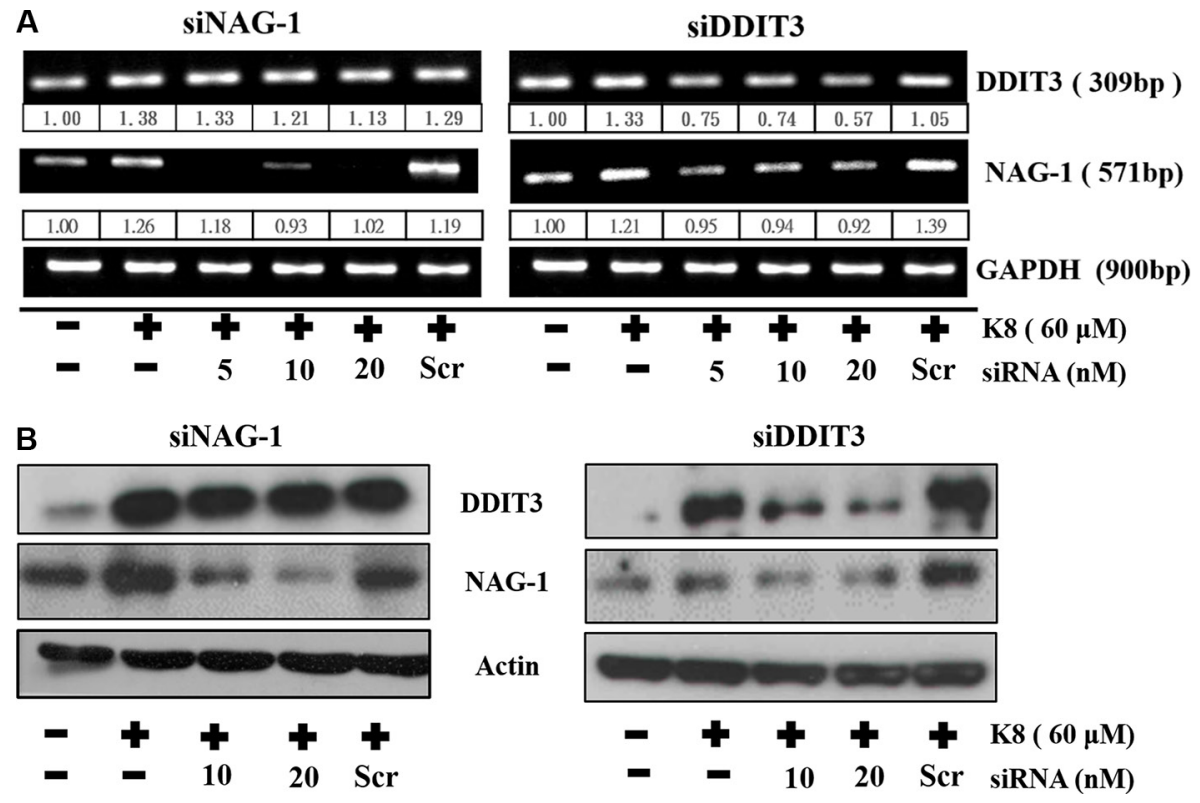

Figure 5: DDIT3 contributes to the regulation of NAG-1 gene expression. (A) RT-PCR analysis of DDIT3 and NAG-1 expression in 8401 were treated with DDIT3 and NAG-1 siRNA. Cells were all transfected with siRNA which targeted DDIT3 or NAG-1 for 6 hrs prior to treatment with K8. (B) Western blot analysis of NAG-1 and DDIT3 in whole cell lysates of 8401 were harvested 24 hrs after treatment with DDIT3 or NAG-1 siRNA. 
a selective inhibitor of the novel isoforms of protein kinase $\mathrm{C} \delta(\mathrm{PKC} \delta)$, significantly induced DDIT3 and NAG-1 expression in HT26 colon carcinoma, but DDIT3 siRNA did not affect rottlerin-induced NAG-1 expression [22].

Previous studies have shown that K8 caused growth inhibition by inducing the G2/M phase arrest of human lung adenocarcinoma and prostate cancer cells, and induced NAG-1 expression considerably [23, 24]. In the cytoskeleton, K8 inhibited tubulin polymerization and its pattern, but the molecular mechanism is not adequately understood [25]. The DDIT3-induced apoptosis via the autophagy pathway was reported in a previous study [26].

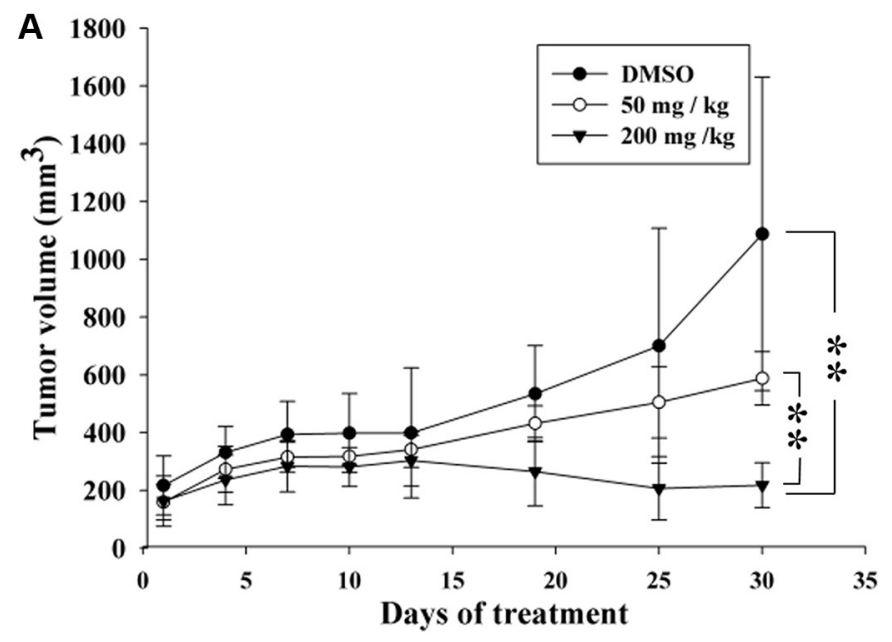

B

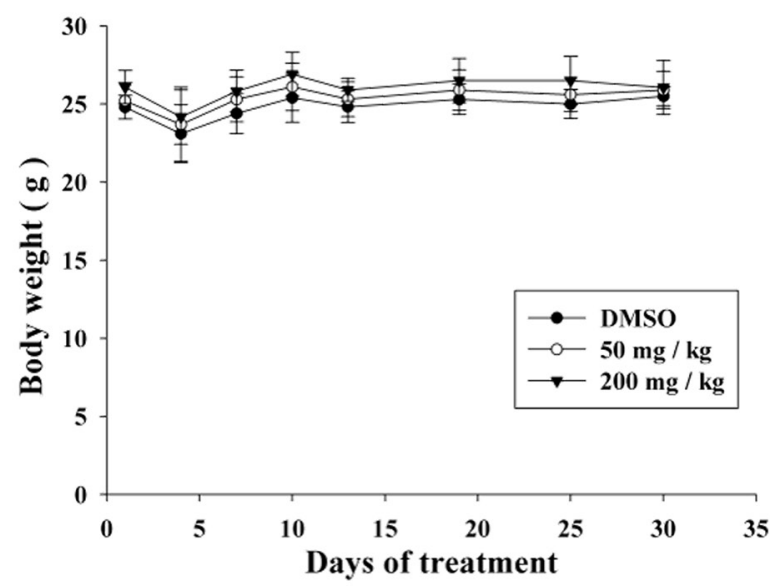

C
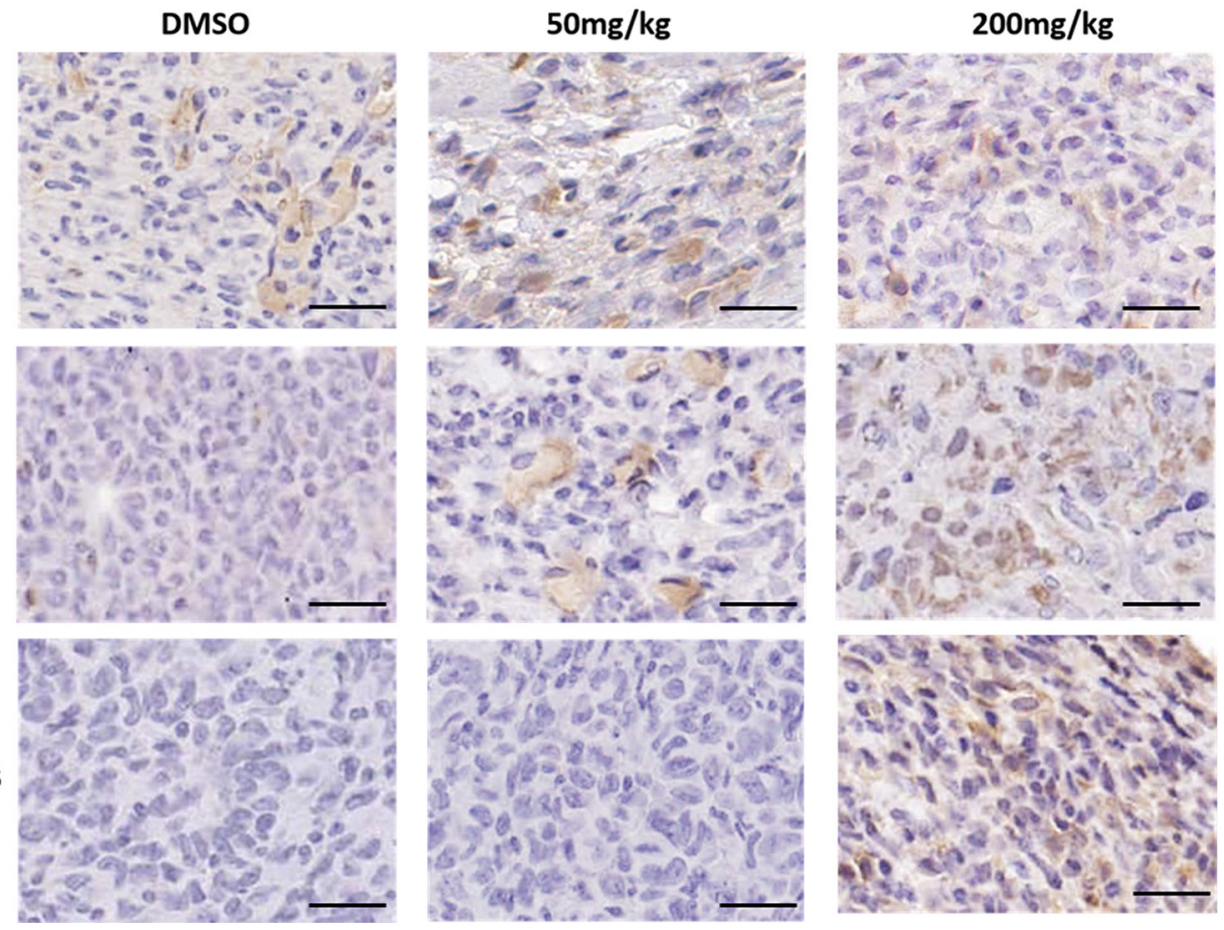

Figure 6: Antitumor effects of $\mathbf{K 8}$ in the nude mice xenograft model. (A) Shown is the tumor growth curve with three different treatment groups: Control (DMSO), $50 \mathrm{mg} / \mathrm{kg}$, and $200 \mathrm{mg} / \mathrm{kg}$ (each group $n=6$ ). (B) Mean body weights for each group during the treatment period. (C) Immunohistochemistry images of DDIT3 and PERK and cleaved caspase3 are staining of tumor sections. Brown coloration indicates apoptotic cells (original magnification $200 \times$ ). Scale bar is $20 \mu \mathrm{m}$. 
glioblastoma stem cell lines, were generous gifts from Dr. Dueng-Yuan Hueng. Roswell Park Memorial Institute (RPMI) 1640 medium, Dulbecco modified Eagle's medium (DMEM), FBS, penicillin, streptomycin, and trypsin-EDTA were purchased from Gibco. Dimethyl sulfoxide, MTT, and Lipofectamine 2000 were purchased from Invitrogen (Carlsbad, CA, USA). Caspase-3 antibody, anti-cleavage caspase-3 antibody, anti-cleavage caspase-7 antibody, anti-cleavage caspase- 9 antibody, DDIT3 antibody, NAG-1 antibody, and monoclonal $\beta$-actin antibodies were purchased from Sigma-Aldrich Co. (St. Louis, MO, USA). PERK antibody and GRP78 antibody were purchased from Genetex. Fluorescein is thiocyanate, Annexin V Apoptosis Detection Kit I, RNA isolation kit, and Polyvinylidene difluoride membranes were purchased from BD Pharmingen.

\section{Cell culture}

Human glioblastoma multiform cell lines were maintained in an RPMI 1640 medium containing 10\% FBS and $100 \mathrm{ng} / \mathrm{mL}$ concentrations of both penicillin and streptomycin. These were maintained at $37^{\circ} \mathrm{C}$ in a humidified atmosphere with $5 \% \mathrm{CO}_{2}$. Glioblastoma stem cell lines 131TXM and 1XM were then maintained in Eagle's minimum essential medium with $10 \%$ FBS and $100 \mathrm{ng} / \mathrm{mL}$ concentrations of both penicillin and streptomycin at $37^{\circ} \mathrm{C}$ in a humidified atmosphere with $5 \%$ $\mathrm{CO}_{2}$. All cultures were free of mycoplasma.

\section{Cell viability assay}

Cells were seeded into 96 well plates at 5,000 cells per well, which were incubated overnight. After incubation for 24 hours, cells were treated with K8 at $0,10,20,40,60,80 \mu \mathrm{M}$ concentrations. Following treatment for 24 hours, we added new medium, containing $5 \mathrm{mg} / \mathrm{ml}$ 3-(4,5-cimethylthiazol-2-yl)-2,5-diphenyl (MTT), which was incubated for 1 hour. We then withdrew the medium and added $100 \mu \mathrm{L}$ of Dimethyl sulfoxide to each well. Absorbance was measured at $595 \mathrm{~nm}$ on a spectrophotometer. All experiments were performed in triplicate.

\section{Cell cycle assay}

Cell cycle progression was assessed via flow cytometry analysis, following DNA staining to quantify the total amount of DNA. Approximately $5 \times 10^{5}$ of 8401 and G2T cells were incubated with $60 \mu \mathrm{M} \mathrm{K} 8$ for the indicated time. The cells were harvested with trypsin-EDTA, collected, washed with PBS, fixed overnight with cold $75 \%$ ethanol, and then stained for 1 hour in the dark with a solution containing $45 \mathrm{mg} / \mathrm{mL}$ of Propidium iodide (PI), $10 \mathrm{mg} / \mathrm{mL}$ of RNase A, and $0.1 \%$ Triton X-100. The cells were then passed through a FACScan flow cytometer, equipped with a $488 \mathrm{~nm}$ argon laser, to measure the DNA content. Cell Quest 3.0.1 (Becton Dickinson; Franklin Lakes, NJ, USA) and ModFitLT 2.0 software were used in order to obtain and analyze data.

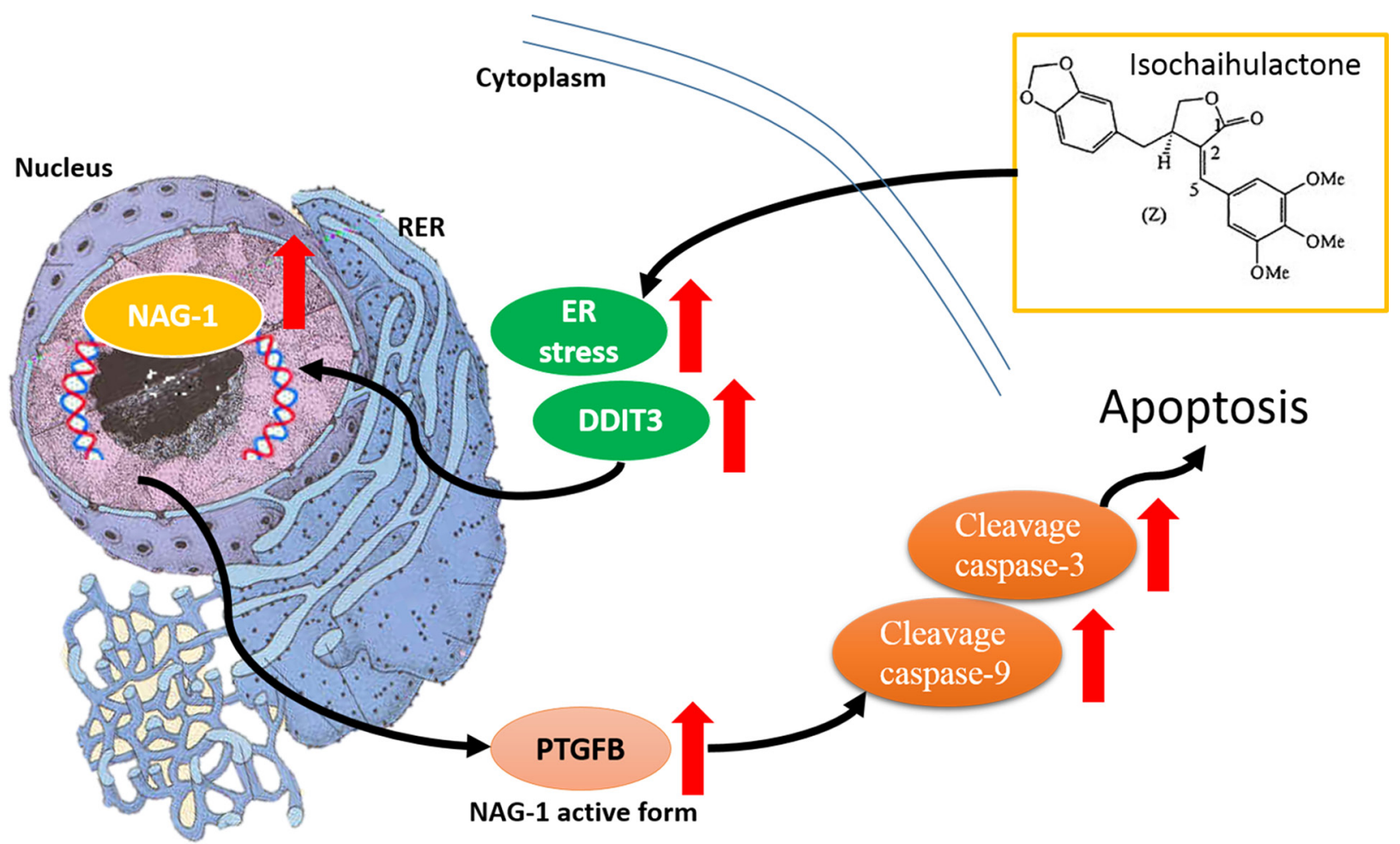

Figure 7: K8 induction of DDIT3 expression which leads to the modulation of NAG-1, and the whole process results in GBM cell apoptosis. Scheme summarizes the K8-induced signaling pathway in glioblastoma cell apoptosis. 


\section{Annexin V and PI double-staining assay}

Apoptosis was analyzed by using the method by van Engeland et al. (1998) to detect the integrity of the cellular membrane and the externalization of phosphatidylserine. Approximately $10^{6}$ cells were grown in 35-mm-diameter plates. The cells were treated with various herbal extracts and chemicals according to the experimental design, and were then labeled with $10 \mu \mathrm{g} / \mathrm{mL}$ of annexinV-FLOUS and $20 \mu \mathrm{g} / \mathrm{mL}$ of PI before harvesting. After labeling, the cells were washed with a binding buffer and harvested via scraping. They were resuspended in a binding buffer at a concentration of $2 \times 10^{5}$ cells $/ \mathrm{mL}$ prior to flow cytometry analysis (FACScan). WinMDI 2.8 software was used to analyze data. The percentage of cells undergoing apoptosis was determined through 3 independent experiments.

\section{RNA extraction, reverse transcription polymerase chain reaction, and quantitative polymerase chain reaction}

For the microarray and reverse transcription polymerase chain reaction (RT-PCR) experiments, human GBM8401 cells were treated with $60 \mu \mathrm{M}$ K8 for 24 hours. RNA was extracted with TRIzol (Invitrogen) and then purified using RNeasy (Qiagen) in accordance with the manufacturer's instructions. Total RNA from the GBM8401 cells was isolated as mentioned. The cDNA was synthesized through the reverse transcription of $2 \mu \mathrm{g}$ of total RNA by using oligo(dT) and SuperScript II RNA reverse transcriptase (Invitrogen). The cDNA was then used as a template to amplify the corresponding DNA fragments through PCR, utilizing 2 sets of synthetic oligonucleotide primers. DNA amplification was performed using PCR with a Thermocycler 2400 (PerkinElmer Life and Analytical Sciences, Boston, MA, USA) under the following conditions: 30 cycles of denaturation at $95^{\circ} \mathrm{C}$ for 45 seconds, annealing at $58^{\circ} \mathrm{C}$ for 45 seconds, and extension at $72^{\circ} \mathrm{C}$ for 15 seconds. The primers used for PCR amplification are listed in Table 2. The PCR products were separated on $2 \%$ agarose gels, stained with ethidium bromide, and visualized using the FluorChem imaging system (Alpha InnoTech, San Leandro, CA, USA). The levels of glyceraldehyde-3phosphate dehydrogenase were used as the control.

\section{Protein extraction and western blotting}

Approximately $2 \times 10^{6}$ cells were cultured in two $100-\mathrm{mm}$ dishes, and then incubated with various concentrations of $\mathrm{K} 8$ for 24 hours. The cells were lysed on ice with $100 \mu \mathrm{L}$ of a lysis buffer $50 \mathrm{mM}$ Tris-HCl, $0.5 \mathrm{M}$ $\mathrm{NaCl}$ at a $\mathrm{pH}$ of $7.5,5 \mathrm{mM} \mathrm{MgCl}, 0.5 \%$ Nonidet P-40, $1 \mathrm{mM}$ phenylmethylsulfonyl fluoride, $1 \mu \mathrm{g} / \mathrm{mL}$ pepstatin, and $50 \mathrm{mg} / \mathrm{mL}$ leupeptin. The cells were then centrifuged at $14,000 \mathrm{~g}$ for 20 minutes at $4{ }^{\circ} \mathrm{C}$. Protein concentrations in supernatants were quantified using a bovine serum albumin protein assay kit. Electrophoresis was performed on a NuPAGE Bis-Tris Electrophoresis System using $50 \mu \mathrm{g}$ of reduced extract per lane. Resolved proteins were then transferred to polyvinylidene difluoride membranes, which were blocked with 5\% nonfat milk for 1 hour and probed overnight with an appropriate dilution of primary antibodies at $4{ }^{\circ} \mathrm{C}$. The membranes were washed 3 times with $0.1 \%$ Tween 20 and incubated with horseradish peroxidase-conjugated secondary antibody at a 1:5000 dilution for 1 hour at room temperature. And we use $\beta$-actin as loading control. The ECL detection system was used in accordance with the manufacturer's instructions.

\section{RNAi transfection}

The DDIT3 siRNA targeting the sequence AGAGGC-AUA-CCA-AGA-UCC-A and NAG-1 siRNA targeting the sequence GAC-UCC-AGA-UUC-CGAGAG-U were synthesized (Ambion, Austin, TX, USA). Cells at $50-60 \%$ confluence were transfected with the transfection reagent lipofectamine 2000 in accordance with the manufacturer's protocol, with DDIT3 or NAG-1 siRNA (Ambion) at concentrations of 5-20 nM for 48 hours. The transfected medium was removed, and the cells were treated with $\mathrm{K} 8$ or a vehicle for up to 48 hours. After incubation, RNA was isolated for RT-PCR, and the cells were collected for apoptosis analysis.

\section{Tumor xenograft model}

Xenograft mice were used as a model system to examine the in vivo cytotoxic effect of $\mathrm{K} 8$, and they were injected subcutaneously with 8401 cells $\left(2 \times 10^{6}\right.$ cells $\left./ \mathrm{mL}\right)$. $\mathrm{BALB} / \mathrm{c}$ Nude (nu/nu) mice were purchased from the National Science Council (Taipei, Taiwan), and all procedures were performed in compliance with the standard operating procedures of the Laboratory Animal Center of Tzu Chi University (Hualien, Taiwan). Animal were housed with an inverse 12 hours day-night cycle with lights on at $8: 30 \mathrm{pm}$ in a temperature $\left(22 \pm 1^{\circ} \mathrm{C}\right)$ and humidity $(55 \pm 5 \%)$ controlled room. Prior to surgery the animal were housed to familiar new environment for 7 days. Experiments were performed using 6-8 week-old mice weighing $23-27 \mathrm{~g}$. When the tumor volume reached 240-260 $\mathrm{mm}^{3}$, the animals were separated randomly into 3 groups: control, low-dose $(50 \mathrm{mg} / \mathrm{kg})$, and high-dose $(200 \mathrm{mg} / \mathrm{kg})$. Each group consisted of 6 mice at Day 0. Daily subcutaneous administrations of $\mathrm{K} 8$, dissolved in DMSO, were performed from Days 0-4 away from the inoculated tumor sites. The control group was treated with DMSO only. The mice were weighed 3 times per week, up to Day 30 , to simultaneously monitor the effects and tumor volume by measuring the length $(\mathrm{L})$ and width $(\mathrm{W})$ of the tumors. The tumor volume at Day $n(\mathrm{TVn})$ was calculated as TV $\left(\mathrm{mm}^{3}\right)=\left(\mathrm{L} \times \mathrm{W}^{2} / 2\right)$. The relative tumor volume 
at Day $n(\mathrm{RTVn})$ versus that at Day 0 was expressed as follows: $\mathrm{RTVn}=\mathrm{TVn} / \mathrm{TV} 0$. Tumor regression $(\mathrm{T} / \mathrm{C}$, in $\%)$ in the treated versus control mice was calculated as follows: $\mathrm{T} / \mathrm{C}(\%)=($ mean RTV of treated group $) /($ mean RTV of control group) $\times 100$. Xenograft tumors, as well as the vital organs of the treated and control mice, were harvested and fixed in $4 \%$ formalin, embedded in paraffin, and cut into 4-mm sections for histological examination.

\section{Immunoblotting}

Each group of subcutaneous GBM 8401 tumors were fixed in $4 \%$ formalin at $4^{\circ} \mathrm{C}$ for 16 hours and then embedded in paraffin. Paraffin sections were deparaffinized with xylene and rehydrated ethanol solutions graded from 75-95\%. Then the sections were incubated with a blocking solution, which contained hydrogen peroxide and protein blocks for $20 \mathrm{~min}$ at room temperature, followed by incubation overnight with anti-DDIT3 rabbit polyclonal antibody in the blocking solution at $4^{\circ} \mathrm{C}$. After that, we used the UltraVision Quanto Detection System (Thermo Scientific, Waltham, MA, USA) to detect the signal. Finally, the sections were counterstained with hematoxylin, mounted, scanned by Aperio Digital Pathology Slide Scanners (Leica Biosystems, CS2) at a magnification of 200, and photographed.

\section{Statistics}

Immunoblot bands and gel bands were being quantitative by ImageJ software (Version 1.6). Statistical analyses were performed with Microsoft Excel 2016. Targeted bands were normalized with $\beta$-actin and GAPDH quantitative value. Data are shown as the mean $\pm \mathrm{SD}$. Statistical differences were analyzed using the Student $t$ test for normally distributed values, and $P$ values $<0.05$ were considered significant. For multiple comparisons, one-way ANOVA was performed, followed by Tukey's range test, and a statistically significant difference was considered when $P<0.05$.

\section{ACKNOWLEDGMENTS AND FUNDING}

We wish to thank Lisa Lin and Wallace Academic Editing office for their support. This study is supported by the Ministry of Science and Technology, Taiwan (MOST 103-2320-B-303-002-MY3) and the Taiwan Ministry of Health and Welfare Clinical Trial and Research Center of Excellence (MOHW105-TDU-B-212-133019) and Health and Welfare Surcharge of Tobacco Products, China Medical University Hospital Cancer Research Center of Excellence (MOHW105-TDU-B-212-134003, Taiwan) and CMU under the Aim for Top University Plan of the Ministry of Education, Taiwan.

\section{CONFLICTS OF INTEREST}

No potential conflicts of interest were disclosed.

\section{REFERENCES}

1. Kato H, Nishitoh H. Stress responses from the endoplasmic reticulum in cancer. Front Oncol. 2015; 5.

2. Schröder M, Kaufman RJ. ER stress and the unfolded protein response. Mutat Res. 2005; 569:29-63.

3. Ron D. Translational control in the endoplasmic reticulum stress response. J Clin Invest. 2002; 110:1383-1388.

4. Martinon F. Targeting endoplasmic reticulum signaling pathways in cancer. Acta Oncol . 2012; 51:822-830.

5. Wang Y, Alam GN, Ning Y, Visioli F, Dong Z, Nör JE, Polverini PJ. The unfolded protein response induces the angiogenic switch in human tumor cells through the PERK/ ATF4 pathway. Cancer Res. 2012; 72:5396-5406.

6. Lee E, Nichols P, Spicer D, Groshen S, Mimi CY, Lee AS. GRP78 as a novel predictor of responsiveness to chemotherapy in breast cancer. Cancer Res. 2006; 66: 7849-7853.

7. Tanimura A, Yujiri T, Tanaka Y, Hatanaka M, Mitani N, Nakamura Y, Mori K, Tanizawa Y. The anti-apoptotic role of the unfolded protein response in Bcr-Abl-positive leukemia cells. Leuk Res. 2009; 33:924-928.

8. Xing X, Lai M, Wang Y, Xu E, Huang Q. Overexpression of glucose-regulated protein 78 in colon cancer. Clin Chim Acta. 2006; 364:308-315.

9. Pyrko P, Schönthal AH, Hofman FM, Chen TC, Lee AS. The unfolded protein response regulator GRP78/BiP as a novel target for increasing chemosensitivity in malignant gliomas. Cancer Res. 2007; 67:9809-9816.

10. Lee HK, Xiang C, Cazacu S, Finniss S, Kazimirsky G, Lemke N, Lehman NL, Rempel SA, Mikkelsen T, Brodie C. GRP78 is overexpressed in glioblastomas and regulates glioma cell growth and apoptosis. Neuro-oncology. 2008; 10:236-243.

11. Prabhu A, Sarcar B, Kahali S, Shan Y, Chinnaiyan P. Targeting the unfolded protein response in glioblastoma cells with the fusion protein EGF-SubA. PLoS One. 2012; 7:e52265.

12. Huynh TT, Lin CM, Lee WH, Wu AT, Lin YK, Lin YF, Yeh CT, Wang LS. Pterostilbene suppressed irradiation-resistant glioma stem cells by modulating GRP78/miR-205 axis. J Nutr Biochem. 2015; 26:466-475.

13. Martin S, Lamb H, Brady C, Lefkove B, Bonner M, Thompson P, Lovat P, Arbiser J, Hawkins A, Redfern C. Inducing apoptosis of cancer cells using small-molecule plant compounds that bind to GRP78. Br J Cancer. 2013; 109:433-443.

14. Tian X, Ye J, Alonso-Basanta M, Hahn SM, Koumenis C, Dorsey JF. Modulation of CCAAT/enhancer binding protein homologous protein (CHOP)-dependent DR5 expression by 
nelfinavir sensitizes glioblastoma multiforme cells to tumor necrosis factor-related apoptosis-inducing ligand (TRAIL). J Biol Chem. 2011; 286:29408-29416.

15. Chen YL, Lin PC, Chen SP, Lin CC, Tsai NM, Cheng YL, Chang WL, Lin SZ, Harn HJ. Activation of nonsteroidal anti-inflammatory drug-activated gene-1 via extracellular signal-regulated kinase 1/2 mitogen-activated protein kinase revealed a isochaihulactone-triggered apoptotic pathway in human lung cancer A549 cells. J Pharmacol Exp Ther. 2007; 323:746-756.

16. Chiu SC, Wang MJ, Yang HH, Chen SP, Huang SY, Chen YL, Lin SZ, Harn HJ, Pang CY. Activation of NAG-1 via JNK signaling revealed an isochaihulactone-triggered cell death in human LNCaP prostate cancer cells. BMC cancer. 2011; 11:1.

17. Moreno JA, Halliday M, Molloy C, Radford H, Verity N, Axten JM, Ortori CA, Willis AE, Fischer PM, Barrett DA. Oral treatment targeting the unfolded protein response prevents neurodegeneration and clinical disease in prioninfected mice. Sci Transl Med. 2013; 5:206ra138-206ra138.

18. Clarke HJ, Chambers JE, Liniker E, Marciniak SJ. Endoplasmic reticulum stress in malignancy. Cancer cell. 2014; 25:563-573.

19. Miyake H, Hara I, Arakawa S, Kamidono S. Stress protein GRP78 prevents apoptosis induced by calcium ionophore, ionomycin, but not by glycosylation inhibitor, tunicamycin, in human prostate cancer cells. J Cell Biochem. 2000; 77:396-408.

20. Booth L, Cazanave SC, Hamed HA, Yacoub A, Ogretmen B, Chen CS, Grant S, Dent P. OSU-03012 suppresses GRP78/BiP expression that causes PERK-dependent increases in tumor cell killing. Cancer Biol Ther. 2012; 13:224-236.

21. Park SH, Choi HJ, Yang H, Do KH, Kim J, Kim HH, Lee H, Oh CG, Lee DW, Moon Y. Two in-and-out modulation strategies for endoplasmic reticulum stress-linked gene expression of pro-apoptotic macrophage-inhibitory cytokine 1. J Biol Chem. 2012; 287:19841-19855.

22. Lim JH, Woo SM, Min KJ, Park EJ, Jang JH, Seo BR, Iqbal T, Lee TJ, Kim SH, Choi YH. Rottlerin induces apoptosis of HT29 colon carcinoma cells through NAG-1 upregulation via an ERK and p38 MAPK-dependent and PKC $\delta$-independent mechanism. Chem Biol Interact. 2012; 197:1-7.

23. Chiu SC, Wang MJ, Yang HH, Chen SP, Huang SY, Chen YL, Lin SZ, Harn HJ, Pang CY. Activation of NAG-1 via JNK signaling revealed an isochaihulactone-triggered cell death in human LNCaP prostate cancer cells. BMC cancer. 2011; 11:146.

24. Ou JP, Lin HY, Su KY, Yu SL, Tseng I, Chen CJ, Hsu HC, Chan DC, Sophia Chen YL. Potential therapeutic role of $\mathrm{Z}$-isochaihulactone in lung cancer through induction of apoptosis via notch signaling. Evid Based Complement Alternat Med. 2012; 2012.

25. Chen YL, Lin SZ, Chang JY, Cheng YL, Tsai NM, Chen SP, Chang WL, Harn HJ. In vitro and in vivo studies of a novel potential anticancer agent of isochaihulactone on human lung cancer A549 cells. Biochem Pharmacol. 2006; 72:308-319.

26. B'chir W, Chaveroux C, Carraro V, Averous J, Maurin AC, Jousse C, Muranishi Y, Parry L, Fafournoux P, Bruhat A. Dual role for $\mathrm{CHOP}$ in the crosstalk between autophagy and apoptosis to determine cell fate in response to amino acid deprivation. Cell Signal. 2014; 26:1385-1391.

27. Zu K, Bihani T, Lin A, Park Y, Mori K, Ip C. Enhanced selenium effect on growth arrest by BiP/GRP78 knockdown in p53-null human prostate cancer cells. Oncogene. 2006; 25:546-554. 